Research Paper

\title{
Dopamine receptor D2 genetic variations is associated with the risk and clinicopathological variables of urothelial cell carcinoma in a Taiwanese population
} \author{
Yang $7,8, \bowtie$, Ming-Hsien Chien $1,9, \square$ \\ 1. Graduate Institute of Clinical Medicine, College of Medicine, Taipei Medical University, Taipei, Taiwan \\ 2. Department of Surgery, Tungs' Taichung Metro Harbor Hospital, Taichung, Taiwan \\ 3. Department of Urology, Wan Fang Hospital, Taipei Medical University, Taipei, Taiwan \\ 4. Department of Urology, School of Medicine, College of Medicine, Taipei Medical University, Taipei, Taiwan \\ 5. Division of Urology, Department of Surgery, Taichung Veterans General Hospital, Taichung, Taiwan \\ 6. School of Medicine, Chung Shan Medical University, Taichung, Taiwan \\ 7. Institute of Medicine, Chung Shan Medical University, Taichung, Taiwan \\ 8. Department of Medical Research, Chung Shan Medical University Hospital, Taichung, Taiwan \\ 9. Department of Medical Education and Research, Wan Fang Hospital, Taipei Medical University, Taipei, Taiwan.
}

Min-Che Tung1,2, Yu-Ching Wen",4, Shian-Shiang Wang,5,6, Yung-Wei Lin³,4, Yu-Cheng Liu1,2, Shun-Fa

$\triangle$ Corresponding authors: Ming-Hsien Chien, PhD, Graduate Institute of Clinical Medicine, Taipei Medical University, 250 Wu-Hsing Street, Taipei 11031, Taiwan; Phone: 886-2-27361661, ext. 3237; Fax: 886-2-27390500; E-mail: mhchien1976@gmail.com or Shun-Fa Yang, PhD, Institute of Medicine, Chung Shan Medical University, 110 Chien-Kuo N. Road, Section 1, Taichung 402, Taiwan; Phone: 886-4-2473959, ext. 34253; Fax: 886-4-24723229; E-mail: ysf@csmu.edu.tw

(C) Ivyspring International Publisher. This is an open access article distributed under the terms of the Creative Commons Attribution (CC BY-NC) license (https://creativecommons.org/licenses/by-nc/4.0/). See http://ivyspring.com/terms for full terms and conditions.

Received: 2018.04.25; Accepted: 2018.06.30; Published: 2018.07.30

\begin{abstract}
Dopamine receptor D2 (DRD2) is overexpressed in several kinds of cancers and was correlated with the prognosis of these cancers. Polymorphisms within the DRD2 gene were shown to be associated with lung and colon cancers. The purpose of this study was to explore effects of DRD2 gene polymorphisms on the susceptibility to and clinicopathological characteristics of urothelial cell carcinoma (UCC). In total, 369 patients diagnosed with UCC and 738 healthy controls were enrolled to analyze DRD2 genotypes at four loci (rs1799732, -141C>del; rs1079597, TaqIB; rs6277, 957C>T; and rs1800497, TaqIA) using a TaqMan-based real-time polymerase chain reaction (PCR). We found a significantly lower risk for UCC in individuals with the DRD2 rs6277 CT genotype compared to those with the wild-type CC genotype (adjusted odds ratio $(A O R)=0.405,95 \%$ confidence interval $(C l): 0.196 \sim 0.837, p=0.015)$. In 124 younger patients (aged < 65 years) of the recruited UCC cohort, patients who carried at least one T allele of DRD2 rs 1800497 were at higher risk $(A O R=2.270,95 \% \mathrm{Cl}: 1.060 \sim 4.860, p=0.033)$ of developing an invasive stage ( $\mathrm{pT} 2 \sim \mathrm{pT} 4)$. In 128 female patients of the recruited UCC cohort, patients who carried at least one deletion allele of DRD2 rs1799732 showed a higher incidence of having an invasive stage $(A O R=2.585,95 \% \mathrm{Cl}$ : 1.066 6.264, $p=0.032)$ and a large tumor (AOR=2.778, 95\% Cl: 1.146 6.735, $p=0.021$ ). Further analyses of The Cancer Genome Atlas (TCGA) and Gene Expression Omnibus (GEO) datasets revealed correlations of the expression of DRD2 with an invasive tumor, tumor metastasis, and the lower survival rate in patients with UCC. Our findings suggest that DRD2 levels might affect the progression of UCC, and the polymorphisms rs6277, rs1800497, and rs1799732 of $D R D 2$ are probably associated with the susceptibility and clinicopathologic development of UCC in a Taiwanese population.
\end{abstract}

Key words: Dopamine receptor D2 gene, Polymorphism, Susceptibility, Clinicopathologic development, Urothelial cell carcinoma

\section{Introduction}

Urothelial cell carcinoma (UCC) is the second leading cause of death among malignancies of the genitourinary tract system in the US. Invasive UCC of the upper urinary tract has a poor prognosis. The 5 -year survival rates of patients with stage T2 and T3 disease are respectively $73 \%$ and $40 \%$, and the median survival for patients with stage T4 disease is only 6 months [1]. There are several types of UCC (bladder, renal pelvis, and ureter carcinomas) with similar histologic features, and approximately $90 \%$ of bladder 
cancers are of the UCC type. In Taiwanese, mortality rates of bladder cancer ranked $13^{\text {th }}$ among all cancer deaths for females and $12^{\text {th }}$ among males [2]. There are many environmental risk factors reported to be correlated with susceptibility to bladder cancer, such as smoking, occupational exposure to aromatic amines, and arsenic exposure in drinking water [3]. In addition, increasing evidence regarding the impacts of genetic factors on the risk or clinicopathologic development of UCC was reported [4-6].

Dopamine, an important neurotransmitter, plays a crucial role in inducing cell growth, motility, and gene expression by activating specific dopamine receptors (DRs). DRs are a class of G-protein-coupled receptors and are important in the nervous system. DRs are divided into the D1-like class (DRD1 and DRD5) and D2-like class (DRD2, DRD3, and DRD4). These receptors work antagonistically to modulate the synthesis of cyclic adenosine monophosphate (cAMP) and activity of protein kinase A (PKA) [7]. Dysregulated dopamine secretion or inactivation of DRs was reported to correlate with various neurological diseases such as schizophrenia and Parkinson's disease [8]. In addition to the traditional function of DRs in neurological diseases, among DRs, DRD2 especially was reported to be overexpressed in different cancer types, including cervical, lung, pituitary, colon, and gastric cancers and to be correlated with poor prognoses of these cancers [7, 9]. Moreover, blocking DRD2 was shown to suppress proliferation and induce apoptosis of both cancer cells and cancer stem cells $[7,10]$. These findings suggest an association between DRD2 and several cancers. Although the expression and the effects of DRD2 inhibition were reported in several types of cancer, the correlation between the expression and genetic alteration of DRD2 with the risk of UCC has not been investigated yet.

Among DNA sequence variations, single-nucleotide polymorphisms (SNPs) are the most common event, and this kind of genetic variation may regulate gene expressions to further affect the susceptibility to diseases such as cancers [11, 12]. A number of SNPs in the gene encoding DRD2 were described. Three SNPs, rs1799732 (DRD2 -141C>del), rs6277 (DRD2 957C>T), and rs1800497 (DRD2 TaqIA), are reported to affect the expression and function of this protein [13, 14]. Moreover, because rs1079597 (DRD2 TaqIB) is closer to the regulatory and structural coding regions ( $5^{\prime}$ region) of the gene, it is believed to play a role in transcriptional regulation [15]. These DRD2 SNPs were reported to be associated with the risk of diseases such as Parkinson's disease [16], colorectal cancer [17], and lung cancer [18]. Moreover, rs1800497 were also reported to be associated with adenoma recurrence in the Polyp Prevention Trial [19] and may increase the risk of smoking among cancer patients [20]. Until now, to the best of our knowledge, the effects of DRD2 gene variants on UCC remain poorly investigated. In this study, we explored the contributions of four DRD2 SNPs, rs1799732, rs1079597, rs6277, and rs1800497, which are respectively located on the promoter, intron 1, exon 7, and 3' untranslated region (UTR) downstream regions, to UCC susceptibility and clinicopathological characteristics.

\section{Materials and methods}

\section{Study subjects, ethics, and consent}

In the current study, 369 UCC patients (241 men and 128 women; mean age $=68.81 \pm 11.77$ years) were consecutively recruited from the Taichung Veterans General Hospital, Taichung, Taiwan. For the control groups, 738 healthy control (482 men and 256 women; mean age $=61.19 \pm 5.60$ years) who had neither self-reported history of cancer of any sites. For the UCC patients, the clinical staging of the UCC patients were staged at the time of diagnosis following the tumor/node/metastasis staging system of the American Joint Committee on Cancer (AJCC) [21]. The study subjects were investigated by interviewer-administered questionnaires containing questions involving demographic characteristics to collect their personal information and characteristics. $37.8 \%$ and $30.4 \%$ of the recruited control subjects and UCC patients had a smoking habit. The study protocol was approved by the Institutional Review Board of Taichung Veterans General Hospital, and the informed written consent was obtained from each individual before the initiation of the study. Whole-blood specimens collected from controls and UCC patients were placed in tubes containing ethylenediaminetetraacetic acid (EDTA), immediately centrifuged, and then stored at $-80^{\circ} \mathrm{C}$.

\section{Genomic DNA extraction and DRD2 polymorphism selection}

To acquire genomic DNA, preserved blood in EDTA anti-coagulated tubes was extracted using a QIAamp DNA Blood Mini Kit (Qiagen, Valencia, CA, USA) according to the manufacturer's protocols. We dissolved DNA in pH 7.8 TE buffer containing $10 \mathrm{mM}$ Tris and $1 \mathrm{mM}$ EDTA and then quantified it by a measurement of OD260. The final preparation was stored at $-20^{\circ} \mathrm{C}$ and was used to act as templates for the PCR. In total, four SNPs in DRD2 were selected from the International HapMap Project data for this study. We included -141C>del (rs1799732) in the promoter region; rs1079597 (TaqIB) and rs6277 
$(957 \mathrm{C}>\mathrm{T})$, which are respectively located in intron 1 and exon 7; and rs1800497 (TaqIA) located downstream of the 3' UTR. We selected these SNPs for this study since these SNPs were reported to affect the expression and binding potential of DRD2 and also correlated with the susceptibility of colorectal and lung cancers $[14,17,18,22]$.

\section{Genotyping of DRD2 SNPs}

Allelic discrimination of DRD2 rs1799732 (assay

ID: C_33641686_10), rs1079597 (assay ID: C__2278884_10), rs6277 (assay ID: C_11339240_10), and rs1800497 (assay ID: C__7486676_10) SNP was assessed using the TaqMan SNP Genotyping Assay with an ABI StepOnePlus ${ }^{\mathrm{TM}}$ Real-Time PCR System (Thermo Fisher Scientific, MA) and further evaluated with SDS version 3.0 software (Applied Biosystems, Foster City, CA) as described previously [23].

\section{Statistical analysis}

Fisher's exact test and the Mann-Whitney U-test were used to evaluate differences in age, gender, and distributions of demographic characteristic between the controls and patients with UCC. The odds ratios (ORs) and 95\% confidence intervals (CIs) of associations of genotype frequencies and clinical pathological characteristics with UCC risk were estimated by multiple logistic regression models. $p<0.05$ was considered statistically significant. Data were analyzed with SAS statistical software (vers. 9.1, 2005; SAS Institute, Cary, NC).

\section{Results}

Demographic and lifestyle characteristics of UCC patients are shown in Table 1. Comparing to the healthy controls, there was no significant difference in the distribution of sex, but significantly different distributions of age $(p<0.001)$ and tobacco use $(p=$ $0.014)$ were found. To reduce the possible interference of confounding variables, adjusted ORs (AORs) with 95\% CIs were estimated by multiple logistic regression models after controlling for other covariates in each comparison.

Genotype distributions of polymorphisms in the promoter region (rs1799732, DRD2 -141C $>$ Del), intron 1 (rs1079597, DRD2 TaqIB), exon 7 (rs6277, DRD2 957C $>$ T), and the 3' UTR downstream (rs1800497, DRD2 TaqIA) between patients and controls are presented in Table 2. Genotypic distributions of DRD2 rs1799732, rs1079597, rs6277, and rs1800497 in the control group conformed to Hardy-Weinberg equilibrium ( $\mathrm{p}=0.846, \chi^{2}$ value: $0.038 ; \mathrm{p}=0.098, \chi^{2}$ value: $2.750 ; p=0.277, \chi^{2}$ value: 1.181 and $p=0.423, \chi^{2}$ value: 0.641 , respectively). The distributions of DRD2 genotypes revealed that the most frequent alleles were heterozygous G/A and C/T for the rs1079597 and rs1800497 loci, respectively, and homozygous Ins/Ins and C/C for the rs1799732 and rs6277 loci, respectively. After adjusting for several variables, we found that subjects with heterozygous $\mathrm{C} / \mathrm{T}$ of the DRD2 rs6277 polymorphism had a 0.405-fold (95\% CI: 0.196 0.837; $p=0.015)$ significantly lower risk of developing UCC compared to those with $\mathrm{C} / \mathrm{C}$ homozygotes. There were no significant differences in genotype distributions between UCC patients and the controls for the rs1799732, rs1079597, or rs1800497 SNPs.

Table 1. Distributions of demographic characteristics of 369 patients with urothelial cell carcinoma (UCC)

\begin{tabular}{ll}
\hline Variable & Patients (N=369) \\
\hline Age (years) & Mean \pm S.D. \\
& $68.81 \pm 11.77$ \\
Gender & \\
Male & $241(65.3 \%)$ \\
Female & $128(34.7 \%)$ \\
Tobacco consumption & \\
No & $257(69.6 \%)$ \\
Yes & $112(30.4 \%)$ \\
Stage & \\
Superficial tumor (pTa or pT1) & $213(57.7 \%)$ \\
Invasive tumor (pT2 pT4) & $156(42.3 \%)$ \\
Tumor T status & \\
T0-T2 & $254(68.8 \%)$ \\
T3-T4 & $115(31.2 \%)$ \\
Lymph node status & \\
N0 & $327(88.6 \%)$ \\
N1+N2 & $42(11.4 \%)$ \\
Metastasis & \\
M0 & $357(96.7 \%)$ \\
M1 & $12(3.3 \%)$ \\
Histopathologic grading & $51(13.8 \%)$ \\
Low grade & $318(86.2 \%)$ \\
High grade & \\
\hline & \\
\hline
\end{tabular}

Impacts of the DRD2 polymorphic genotype on the clinicopathological development of UCC including clinical statuses of cancer stage, primary tumor size, lymph node involvement, distant metastasis, and histologic grade were further evaluated, and results are shown in Tables 3 and 4 . We classified UCC patients into two subgroups, including patients who had the homozygous wild-type (WT) alleles or who had at least one polymorphic allele. Among 124 younger UCC patients under 65 years of age, those carrying at least 1 polymorphic $\mathrm{T}$ allele of DRD2 rs1800497showed a significantly higher risk of having a muscle-invasive tumor (OR=2.270, 95\% CI: 1.060 4.860, $p=0.033$ ) than those carrying the WT gene (Table 3). Moreover, DRD2 rs1799732 promoter polymorphisms presented significant differences with clinical stage $(\mathrm{OR}=2.585$, 95\% CI: 1.066 6.264, $p=0.032$ ) and tumor size $(\mathrm{OR}=2.778,95 \% \mathrm{CI}: 1.146 \sim 6.735, p=0.021)$ in female UCC patients with at least one DRD2-141Del polymorphism (Table 4). 
Table 2. Distribution frequency of DRD2 genotypes in 738 controls and 369 UCC patients.

\begin{tabular}{|c|c|c|c|c|c|}
\hline Location & Variable & Controls $(N=738) n(\%)$ & Patients $(N=369) n(\%)$ & OR $(95 \% \mathrm{CI})$ & AOR $(95 \% \mathrm{CI})$ \\
\hline \multirow{7}{*}{ Promoter } & rs1799732 & & & & \\
\hline & $-141 C>$ del & & & & \\
\hline & Ins/Ins & $597(80.9 \%)$ & $303(82.1 \%)$ & 1.00 & 1.00 \\
\hline & Ins/Del & $133(18.0 \%)$ & $62(16.8 \%)$ & $0.918(0.659 \sim 1.280)$ & $0.642(0.383 \sim 1.076)$ \\
\hline & Del/Del & $8(1.1 \%)$ & $4(1.1 \%)$ & $0.985(0.294 \sim 3.298)$ & $1.067(0.209 \sim 5.441)$ \\
\hline & Ins/Del + Del/Del & $141(19.1 \%)$ & $66(17.9 \%)$ & $0.922(0.667 \sim 1.274)$ & $0.665(0.403 \sim 1.095)$ \\
\hline & rs1079597 & & & & \\
\hline \multirow[t]{6}{*}{ Intron 1} & TaqIB & & & & \\
\hline & GG & $239(32.4 \%)$ & $131(35.5 \%)$ & 1.00 & 1.00 \\
\hline & GA & $381(51.6 \%)$ & $172(46.6 \%)$ & $0.824(0.623 \sim 1.089)$ & $0.969(0.655 \sim 1.433)$ \\
\hline & $\mathrm{AA}$ & $118(16.0 \%)$ & $66(17.9 \%)$ & $1.020(0.706 \sim 1.476)$ & $1.138(0.686 \sim 1.888)$ \\
\hline & $\mathrm{GA}+\mathrm{AA}$ & $499(67.6 \%)$ & $238(64.5 \%)$ & $0.870(0.669 \sim 1.132)$ & $1.010(0.698 \sim 1.463)$ \\
\hline & rs6277 & & & & \\
\hline \multirow[t]{5}{*}{ Exon 7} & $957 \mathrm{C}>\mathrm{T}$ & & & & \\
\hline & $\mathrm{CC}$ & $646(87.5 \%)$ & $339(91.9 \%)$ & 1.00 & 1.00 \\
\hline & $\mathrm{CT}$ & $87(11.8 \%)$ & $30(8.1 \%)$ & $\begin{array}{l}0.657(0.425 \sim 1.016) \\
p=0.059\end{array}$ & $\begin{array}{l}0.405(0.196 \sim 0.837)^{*} \\
p=0.015\end{array}$ \\
\hline & TT & $5(0.7 \%)$ & $0(0 \%)$ & 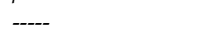 & 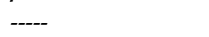 \\
\hline & $\mathrm{CT}+\mathrm{TT}$ & $92(12.5 \%)$ & $30(8.1 \%)$ & $\begin{array}{l}0.621(0.403 \sim 0.958)^{*} \\
p=0.030\end{array}$ & $\begin{array}{l}0.385(0.187 \sim 0.794)^{*} \\
p=0.010\end{array}$ \\
\hline \multirow{6}{*}{$\begin{array}{l}\text { 3' UTR } \\
\text { downstream }\end{array}$} & rs1800497 & & & & \\
\hline & TaqIA & & & & \\
\hline & $\mathrm{CC}$ & $276(37.4 \%)$ & $146(39.6 \%)$ & 1.00 & 1.00 \\
\hline & $\mathrm{CT}$ & $359(48.6 \%)$ & $162(43.9 \%)$ & $0.853(0.649 \sim 1.121)$ & $0.971(0.661 \sim 1.426)$ \\
\hline & TT & $103(14.0 \%)$ & $61(16.5 \%)$ & $1.120(0.770 \sim 1.629)$ & $1.384(0.839 \sim 2.282)$ \\
\hline & $\mathrm{CT}+\mathrm{TT}$ & $462(62.6 \%)$ & $223(60.4 \%)$ & $0.912(0.706 \sim 1.179)$ & $1.066(0.744 \sim 1.527)$ \\
\hline
\end{tabular}

The odds ratio (OR) with their $95 \%$ confidence intervals (CIs) were estimated by logistic regression models.

The adjusted OR (AOR) with their 95\% CIs were estimated by multiple logistic regression models after controlling for age, sex, and tobacco consumption.

Table 3. Distribution frequency of the clinical status and DRD2 rs 1800497 genotype frequencies in 124 younger patients with UCC (aged $<65$ years)

\begin{tabular}{|c|c|c|c|c|}
\hline & DRD2 (rs1800497) & & & \\
\hline Variable & $\mathrm{CC}(\%)(n=45)$ & CT+TT $(\%)(n=79)$ & OR $(95 \%$ CI $)$ & $p$ value \\
\hline \multicolumn{5}{|l|}{ Stage } \\
\hline Superficial tumor (pTa or pT1) & $30(66.7 \%)$ & $37(46.8 \%)$ & 1.00 & \\
\hline Invasive tumor (pT2 pT4) & $15(33.3 \%)$ & $42(53.2 \%)$ & $2.270(1.060 \sim 4.860)$ & $p=0.033^{*}$ \\
\hline \multicolumn{5}{|l|}{ Tumor T status } \\
\hline $\mathrm{T} 0 \sim \mathrm{T} 2$ & $33(73.3 \%)$ & $52(65.8 \%)$ & 1.00 & \\
\hline $\mathrm{T} 3$ or $\mathrm{T} 4$ & $12(26.7 \%)$ & $27(34.2 \%)$ & $1.428(0.637 \sim 3.203)$ & $p=0.386$ \\
\hline \multicolumn{5}{|l|}{ Lymph node status } \\
\hline No & $38(84.4 \%)$ & $66(83.5 \%)$ & 1.00 & \\
\hline $\mathrm{N} 1+\mathrm{N} 2$ & $7(15.6 \%)$ & $13(16.5 \%)$ & $1.069(0.393 \sim 2.912)$ & $p=0.896$ \\
\hline \multicolumn{5}{|l|}{ Metastasis } \\
\hline M0 & $45(100 \%)$ & $75(94.9 \%)$ & 1.00 & \\
\hline M1 & $0(0 \%)$ & $4(5.1 \%)$ & --- & $p=0.125$ \\
\hline \multicolumn{5}{|l|}{ Histopathologic grading } \\
\hline Low grade & $8(17.8 \%)$ & $9(11.4 \%)$ & 1.00 & \\
\hline High grade & $37(82.2 \%)$ & $70(88.6 \%)$ & $1.682(0.599 \sim 4.722)$ & $p=0.320$ \\
\hline
\end{tabular}

Table 4. Distribution frequency of the clinical status and DRD2 rs 1799732 genotype frequencies in 128 female patients with UCC.

\begin{tabular}{|c|c|c|c|c|}
\hline & DRD2 (rs1799732) & & & \\
\hline Variable & Ins/Wt (\%) (n=102) & $\begin{array}{l}\text { Ins/Del + Del/Del } \\
(\%)(n=26)\end{array}$ & OR $(95 \% \mathrm{CI})$ & $\mathrm{p}$ value \\
\hline \multicolumn{5}{|l|}{ Stage } \\
\hline Superficial tumor (pTa or $\mathrm{pT} 1$ ) & $63(61.8 \%)$ & $10(38.5 \%)$ & 1.00 & \\
\hline Invasive tumor (pT2 pT4) & $39(38.2 \%)$ & $16(61.5 \%)$ & $2.585(1.066-6.264)$ & $p=0.032 *$ \\
\hline \multicolumn{5}{|l|}{ Tumor $\mathrm{T}$ status } \\
\hline $\mathrm{T} 0 \sim \mathrm{T} 2$ & $75(73.5 \%)$ & $13(50.0 \%)$ & 1.00 & \\
\hline $\mathrm{T} 3$ or $\mathrm{T} 4$ & $27(26.5 \%)$ & $13(50.0 \%)$ & $2.778(1.146-6.735)$ & $p=0.021 *$ \\
\hline \multicolumn{5}{|l|}{ Lymph node status } \\
\hline N0 & $93(91.2 \%)$ & $22(84.6 \%)$ & 1.00 & \\
\hline $\mathrm{N} 1+\mathrm{N} 2$ & $9(8.8 \%)$ & $4(15.4 \%)$ & $1.879(0.530-6.664)$ & $p=0.323$ \\
\hline \multicolumn{5}{|l|}{ Metastasis } \\
\hline M0 & $100(98.0 \%)$ & $25(96.2 \%)$ & 1.00 & \\
\hline M1 & $2(2.0 \%)$ & $1(3.8 \%)$ & $2.000(0.174-22.949)$ & $p=0.571$ \\
\hline \multicolumn{5}{|l|}{ Histopathologic grading } \\
\hline Low grade & $12(11.8 \%)$ & $2(7.7 \%)$ & 1.00 & \\
\hline High grade & $90(88.2 \%)$ & $24(92.3 \%)$ & $1.600(3.335-7.639)$ & $p=0.553$ \\
\hline
\end{tabular}


A

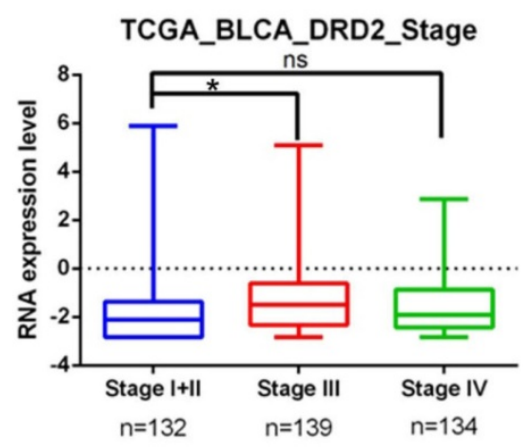

C

TCGA_BLCA_DRD2_pathologic T

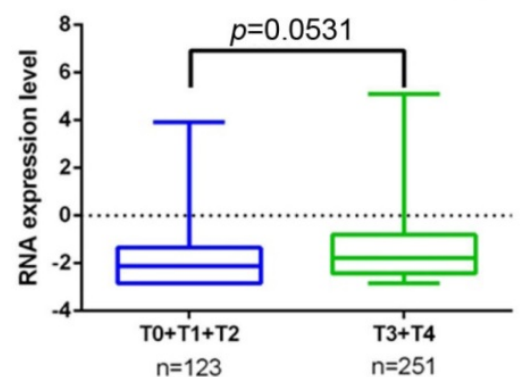

E

DRD2_GSE13507_Invasiveness

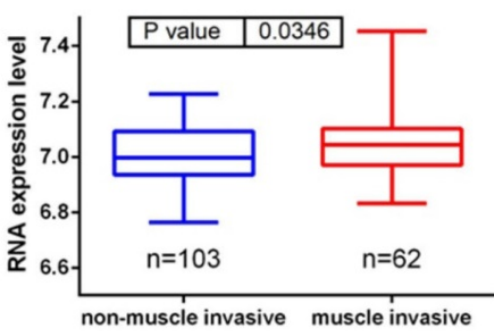

B

TCGA_BLCA_DRD2_pathologic M

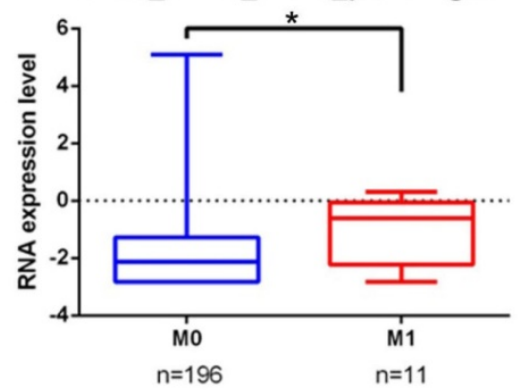

D

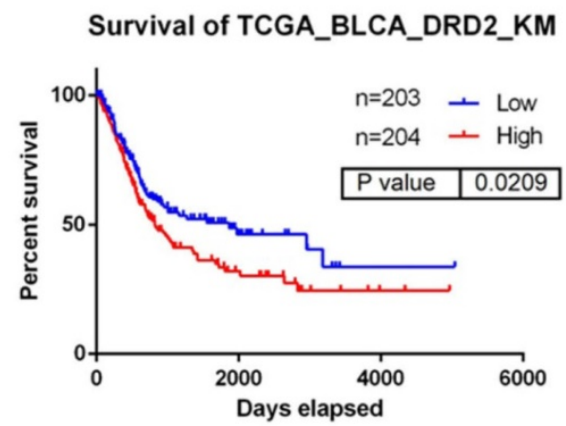

F

Survival of DRD2_GSE13507_KM

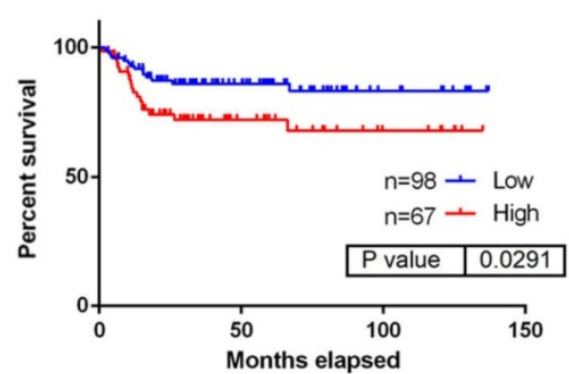

Fig. 1. Clinical relevance of dopamine receptor D2 (DRD2) levels in bladder urothelial cell carcinoma (UCC) patients obtained from TCGA and GEO databases. A-C: DRD2 gene expression levels in bladder urothelial carcinoma tissues from TCGA were compared according to clinical stages (A), Tumor size status (T stages) (C), and metastasis status ( $M$ stages) (B). Statistical significance was analyzed by a $t$-test. * $p<0.05$. D: An overall survival curve was produced for patients with high (red lines) and low (blue lines) DRD2 expression levels using the Kaplan-Meier analysis. p values were determined using a log-rank test. E: Another UCC cohort from the GEO (GSE13507) was analyzed for the correlation between DRD2 expression levels and tumor invasive status. F: A Kaplan-Meier plot of disease-specific survival of 165 patients with bladder UCC (GSE13507) stratified by DRD2 expression. A log-rank test was used to examine between-group differences.

To further dissect the clinical significance of DRD2 in UCC, bladder UCC cases were analyzed from TCGA dataset. Significantly higher DRD2 transcripts were observed in tumors of patients with advanced clinical stage (stage III) and tumor metastasis compared to patients with early stages (stage I+II) and without tumor metastasis $(p<0.05$; Fig. 1A, B). Moreover, relative levels of DRD2 transcripts were higher in patients with larger tumors (T3-T4 status) than in patients with smaller tumors (T0-T2 status) in a marginal trend toward significance $(p=0.053$; Fig. 1C). The Kaplan-Meier plot revealed poor overall survival of patients with high DRD2 expression ( $p=0.0209$; Fig. 1D). Furthermore, 165 bladder UCC cases were also analyzed from the Gene Expression Omnibus (GEO) database (GSE13507), and we found that significantly higher DRD2 was observed in tumors with muscle invasion compared to non-muscle-invasive tumors ( $p=0.0346$; Fig. 1E). Most importantly, patients who had DRD2high tumors had shorter disease-specific survival times compared with those who had DRD2 ${ }^{\text {low }}$ tumors $(p=0.0291$; Fig. 1F). Taken together, the above clinical data indicated that upregulation of DRD2 is a critical event in promoting UCC progression and suggest that DRD2 variants may alter DRD2 expression and subsequently modulate UCC progression. 


\section{Discussion}

There are several reports indicating elevated DRD2 expression in cancer cells compared to normal cells in several cancer types, and DRD2 overexpression correlates with an advanced stage and stemness of cancer and a poor prognosis of patients [7, 9, 24]. Moreover, antagonizing DRD2 signaling either by DRD2 antagonists, such as thioridazine and trifluoperazine, or genetic depletion show the proapoptotic, antimetastatic, antiangiogenic, and anticancer stem cell properties in several tumor types $[7,10,25]$. At present, DRD2 genetic variants have been reported to affect the expression and function of DRD2 and to be correlated with the susceptibility and clinicopathologic development of cancers such as colon and lung cancers, and pituitary adenomas [17, $18,22,26]$. The present study, for the first time to our knowledge, investigated the occurrence of the rs1800497 (TaqIA), rs1079597 (TaqIB), rs6277 (957C > T), and rs1799732 (-141C >Del) polymorphisms of the DRD2 gene in a Taiwanese population, and their possible clinical relevance in patients with UCC. Moreover, we also analyzed the prognostic significance of DRD2 in UCC using the GEO and TCGA databases.

As to the correlation between DRD2 SNPs and risk of UCC, we observed that the rs6277 C/T heterozygous polymorphism was associated with a significantly lower risk of developing UCC compared to the rs6277 C/C homozygous polymorphism. This result suggested that the DRD2 957T allele is a protective factor in UCC. The $957 \mathrm{C}>\mathrm{T}$ polymorphism is located in the exon 7 region. Although this SNP codes for the silent change (proline to proline) at codon 319 , it might still play a role in messenger (m)RNA stability. It was reported that the $\mathrm{T}$ allele variant is associated with alterations of mRNA folding, and decreases in mRNA stability and translation in vitro [22]. Moreover, Hirvonen et al. further indicated that subjects carrying the 957T allele had markedly lower striatal DRD2 availability (binding potential, BP) [27]. We speculated that the rs6277 $\mathrm{T}$ allele, associated with lower DDR2 expression, may influence the tumorigenesis of UCC. Actually, a DRD2 expression analysis in bladder cancer from TCGA and GEO databases revealed that patients who had high DRD2-expressing tumors had shorter overall and disease-specific survival times and advanced clinical stage and metastatic tumors compared to those with low DRD2-expressing tumors. These results indicated that the DRD2 expression level might affect the progression of UCC.

Moreover, our results showed that female individuals carrying at least one DRD2 -141Del polymorphism had a higher risk of an advanced clinical stage and a larger tumor. This DRD2 rs1799732 polymorphism was also reported to be associated with recurrence of adenomas and risk of colon and lung cancers [17-19]. The DRD2 rs1799732 polymorphism comprises a C deletion in the $5^{\prime}$ UTR of DRD2, is considered a functional polymorphism, and is associated with a $20 \% \sim 40 \%$ reduction in basal levels of receptor expression [28]. However, this result was not further demonstrated in a subsequent study [29]. Hence, the influence of the DRD2 rs1799732 polymorphism on DRD2 expression should be further investigated in the future. Furthermore, our results showed that individuals under the age of 65 years carrying at least one polymorphic T allele of DRD2 rs1800497 had a higher risk of having muscle-invasive tumors. The rs1800497 TT genotype was also reported to be associated with advanced adenoma recurrence in the Polyp Prevention Trial [19]. The rs1800497 SNP is $10 \mathrm{~kb}$ downstream from the DRD2 gene and is located in the neighboring ankyrin repeat and kinase domain containing 1 (ANKK1) gene where it causes an amino acid substitution (Glu713Lys) [30]. A previous study indicated that the T allele of rs 1800497 was associated with reduced BP of DRD2 in healthy subjects. In contrast to healthy subjects, the $\mathrm{T}$ allele was associated with increased BP of DRD2 in patients with a major depressive disorder [31]. The effect of rs1800497 on DRD2 binding in UCC patients versus healthy subjects remains unclear and is worthy of further investigation. Classically, the dopamine-DRD2 axis was thought of in terms of antagonism of cAMP-dependent signaling, where $G_{a i}$ subunits bind to and inhibit adenylyl cyclases and further suppress cAMP production and PKA activation [32]. The cAMP/PKA signaling pathway was reported to inhibit the invasive ability of bladder cancer cells [33], suggesting that DDR2 SNPs might affect the dopamine-DRD2 axis to further regulate its downstream cAMP/PKA signaling and modulate invasion of UCC.

It is well-known that smoking is one of the important risk factors for developing bladder cancer [34]. Previous SNP-related studies in UCC also showed significantly higher ratios of individuals with smoking behaviors than in the control group [35]. However, our study was not suitable for investigating the combined effect of tobacco carcinogen exposure and DRD2 SNPs on UCC susceptibility due to a bias in the ratio of individuals with a smoking habit among our recruited UCC patients. In our recruited cohort, there was a higher ratio of individuals with a smoking habit in the healthy control group than in the UCC group. Thus, more UCC patients with a smoking habit should be recruited to further explore the combined effect of DRD2 SNPs and tobacco 
carcinogen exposure on the susceptibility and progression of UCC.

In conclusion, in a Taiwanese population, we first identified that the DRD2 rs6277C/T genotype appeared to attenuate the risk of developing UCC. Female UCC patients harboring at least one DRD2 -141Del polymorphism more easily developed an advanced stage and larger tumors. UCC patients under the age of 65 years carrying at least one polymorphic T allele of DRD2 rs1800497 had a higher risk of developing muscle-invasive tumors. Ultimately, we propose that future work should focus on investigating the functional activities of these polymorphisms mentioned above and their effects on tumor progression, which would help us understand the underlying mechanisms of UCC development.

\section{Acknowledgments}

This study was supported by grant number TTM-TMU-106-01 from Tungs' Taichung Metro Harbor Hospital.

\section{Competing Interests}

The authors have declared that no competing interest exists.

\section{References}

1. Hall MC, Womack S, Sagalowsky AI, Carmody T, Erickstad MD, Roehrborn CG. Prognostic factors, recurrence, and survival in transitional cell carcinoma of the upper urinary tract: a 30-year experience in 252 patients. Urology. 1998; 52: 594-601.

2. Hung CF, Yang CK, Ou YC. Urologic cancer in Taiwan. Jpn J Clin Oncol. 2016; 46: 605-9.

3. Letasiova S, Medve'ova A, Sovcikova A, Dusinska M, Volkovova K, Mosoiu C, et al. Bladder cancer, a review of the environmental risk factors. Environ Health. 2012; 11 Suppl 1: S11.

4. Zhang L, Zhang M, Wang H, Wang Y, Zhou J, Hao Z, et al. Comprehensive Review of Genetic Association Studies and Meta-Analysis on polymorphisms in microRNAs and Urological Neoplasms Risk. Sci Rep. 2018; 8: 3776.

5. Zhang X, Zhang Y. Bladder Cancer and Genetic Mutations. Cell Biochem Biophys. 2015; 73: 65-9.

6. Tung MC, Hsieh MJ, Wang SS, Yang SF, Chen SS, Wang SW, et al. Associations of VEGF-C genetic polymorphisms with urothelial cell carcinoma susceptibility differ between smokers and non-smokers in Taiwan. PLoS One. 2014; 9: e91147.

7. Roney MSI, Park SK. Antipsychotic dopamine receptor antagonists, cancer, and cancer stem cells. Arch Pharm Res. 2018.

8. Birtwistle J, Baldwin D. Role of dopamine in schizophrenia and Parkinson's disease. Br J Nurs. 1998; 7: 832-834, 836, 838-41.

9. Mu J, Huang W, Tan Z, Li M, Zhang L, Ding O, et al. Dopamine receptor D2 is correlated with gastric cancer prognosis. Oncol Lett. 2017; 13: 1223-7.

10. Sachlos E, Risueno RM, Laronde S, Shapovalova Z, Lee JH, Russell J, et al. Identification of drugs including a dopamine receptor antagonist that selectively target cancer stem cells. Cell. 2012; 149: 1284-97.

11. Hua KT, Liu YF, Hsu CL, Cheng TY, Yang CY, Chang JS, et al. 3'UTR polymorphisms of carbonic anhydrase IX determine the miR-34a targeting efficiency and prognosis of hepatocellular carcinoma. Sci Rep. 2017; 7: 4466.

12. Sripichai $\mathrm{O}$, Fucharoen $\mathrm{S}$. Genetic polymorphisms and implications for human diseases. J Med Assoc Thai. 2007; 90: 394-8.

13. Zhang S, Zhang J. The Association of DRD2 with Insight Problem Solving. Front Psychol. 2016; 7: 1865.

14. Doehring A, Hentig N, Graff J, Salamat S, Schmidt M, Geisslinger G, et al. Genetic variants altering dopamine D2 receptor expression or function modulate the risk of opiate addiction and the dosage requirements of methadone substitution. Pharmacogenet Genomics. 2009; 19: 407-14.

15. Hauge XY, Grandy DK, Eubanks JH, Evans GA, Civelli O, Litt M. Detection and characterization of additional DNA polymorphisms in the dopamine D2 receptor gene. Genomics. 1991; 10: 527-30.
16. Dai D, Wang Y, Wang L, Li J, Ma O, Tao J, et al. Polymorphisms of DRD2 and DRD3 genes and Parkinson's disease: A meta-analysis. Biomed Rep. 2014; 2: 275-81.

17. Gemignani F, Landi S, Moreno V, Gioia-Patricola L, Chabrier A, Guino E, et al. Polymorphisms of the dopamine receptor gene DRD2 and colorectal cancer risk. Cancer Epidemiol Biomarkers Prev. 2005; 14: 1633-8.

18. Campa D, Zienolddiny $\mathrm{S}$, Lind $\mathrm{H}$, Ryberg D, Skaug V, Canzian F, et al. Polymorphisms of dopamine receptor/transporter genes and risk of non-small cell lung cancer. Lung Cancer. 2007; 56: 17-23.

19. Murphy G, Cross AJ, Sansbury LS, Bergen A, Laiyemo AO, Albert PS, et al. Dopamine D2 receptor polymorphisms and adenoma recurrence in the Polyp Prevention Trial. Int J Cancer. 2009; 124: 2148-51.

20. Gordiev M, Engstrom PF, Khasanov R, Moroshek A, Sitdikov R, Dgavoronkov $\mathrm{V}$, et al. Genetic analysis of polymorphisms in dopamine receptor and transporter genes for association with smoking among cancer patients. Eur Addict Res.2013;19:105-11.

21. Cheng L, Montironi R, Davidson DD, Lopez-Beltran A. Staging and reporting of urothelial carcinoma of the urinary bladder. Mod Pathol. 2009; 22 Suppl 2:S70-95.

22. Duan J, Wainwright MS, Comeron JM, Saitou N, Sanders AR, Gelernter J, et al. Synonymous mutations in the human dopamine receptor D2 (DRD2) affect mRNA stability and synthesis of the receptor. Hum Mol Genet. 2003; 12: 205-16.

23. Lee HL, Cheng HL, Liu YF, Chou MC, Yang SF, Chou YE. Functional genetic variant of WW domain-containing oxidoreductase (WWOX) gene is associated with hepatocellular carcinoma risk. PLoS One. 2017; 12: e0176141.

24. Mao M, Yu T, Hu J, Hu L. Dopamine D2 receptor blocker thioridazine induces cell death in human uterine cervical carcinoma cell line SiHa. J Obstet Gynaecol Res. 2015; 41: 1240-5.

25. Pulkoski-Gross A, Li J, Zheng C, Li Y, Ouyang N, Rigas B, et al. Repurposing the antipsychotic trifluoperazine as an antimetastasis agent. Mol Pharmacol. 2015; 87: 501-12.

26. Peculis R, Balcere I, Rovite V, Megnis K, Valtere A, Stukens J, et al. Polymorphisms in MEN1 and DRD2 genes are associated with the occurrence and characteristics of pituitary adenomas. Eur J Endocrinol. 2016; 175: 145-53.

27. Hirvonen M, Laakso A, Nagren K, Rinne JO, Pohjalainen T, Hietala J. C957T polymorphism of the dopamine D2 receptor (DRD2) gene affects striatal DRD2 availability in vivo. Mol Psychiatry. 2004; 9: 1060-1.

28. Arinami T, Gao M, Hamaguchi H, Toru M. A functional polymorphism in the promoter region of the dopamine D2 receptor gene is associated with schizophrenia. Hum Mol Genet. 1997; 6: 577-82.

29. Ritchie T, Noble EP. Association of seven polymorphisms of the D2 dopamine receptor gene with brain receptor-binding characteristics. Neurochem Res. 2003; 28: 73-82.

30. Neville MJ, Johnstone EC, Walton RT. Identification and characterization of ANKK1: a novel kinase gene closely linked to DRD2 on chromosome band 11q23.1. Hum Mutat. 2004; 23: 540-5.

31. Savitz J, Hodgkinson CA, Martin-Soelch C, Shen PH, Szczepanik J, Nugent AC, et al. DRD2/ANKK1 Taq1A polymorphism (rs1800497) has opposing effects on D2/3 receptor binding in healthy controls and patients with major depressive disorder. Int J Neuropsychopharmacol. 2013; 16: 2095-101.

32. Bonci A, Hopf FW. The dopamine D2 receptor: new surprises from an old friend. Neuron. 2005; 47: 335-8.

33. Ou Y, Zheng X, Gao Y, Shu M, Leng T, Li Y, et al. Activation of cyclic AMP/PKA pathway inhibits bladder cancer cell invasion by targeting MAP4-dependent microtubule dynamics. Urol Oncol. 2014; 32: 47 e21-48.

34. Hemelt M, Yamamoto H, Cheng KK, Zeegers MP. The effect of smoking on the male excess of bladder cancer: a meta-analysis and geographical analyses. Int J Cancer. 2009; 124: 412-9.

35. Wang YH, Chiou HY, Lin CT, Hsieh HY, Wu CC, Hsu CD, et al. Association between survivin gene promoter $-31 \mathrm{C} / \mathrm{G}$ polymorphism and urothelial carcinoma risk in Taiwanese population. Urology. 2009; 73: 670-4. 